\title{
The topographic relationships between thoracic duct and bronchial arteries and surgical relevance
}

\author{
Nabil Eid $\cdot$ Yuko Ito $\cdot$ Yoshinori Otsuki
}

Received: 8 September 2014 / Accepted: 8 October 2014 / Published online: 29 October 2014

(C) The Japan Esophageal Society and Springer Japan 2014

\section{Dear Editor}

We read with interest the recent paper by Kajiyama et al.: relational topographical anatomy between right bronchial artery and thoracic duct, which has been recently published in Esophagus. Based on operative techniques, the authors studied the relationships between bronchial arteries and thoracic duct, which may be of importance for prevention of chylothorax during surgery of esophageal cancer. They mentioned that they found two new relational anatomical rules between the two vessels: the thoracic duct is posterior to the right bronchial artery on branching abnormally from the descending aorta, however the duct is ventral to the bronchial artery on arising from the third intercostal artery [1].

A few years ago and based on cadaveric dissection, we published two case reports showing the above-mentioned two patterns of thoracic duct and bronchial arteries relationships investigated by those authors. In one case, the thoracic duct passed upward through a vascular tunnel formed by the bifurcated right posterior intercostal artery, then ascended deep to the anomalous (ectopic) common bronchial artery trunk arising from the distal aortic arch and terminating as right and left bronchial arteries. At the latter location, the left recurrent laryngeal nerve looped under the aortic arch superficial to both the thoracic duct and the variant common bronchial artery trunk. Therefore, there is a possibility of injury of both recurrent laryngeal nerve and the thoracic duct during ligation or selective

N. Eid $(\varangle) \cdot$ Y. Ito $\cdot$ Y. Otsuki

Division of Life Sciences, Department of Anatomy and Cell

Biology, Osaka Medical College, 2-7 Daigaku-machi, Takatsuki, Osaka 569-8686, Japan

e-mail: nabil@art.osaka-med.ac.jp; nabileidm@yahoo.com embolization of this artery for the treatment of recurrent hemoptysis, bronchial arteriovenous malformations in addition to surgical treatment of esophageal carcinoma [2]. In the second case, we found a thoracic duct passing ventral and medial to the right bronchial artery arising from the third posterior intercostal artery associated with ectopic common bronchial artery trunk [3, 4]. In the latter papers, diagrams showing such relationships between the thoracic duct and right bronchial artery in addition to the left recurrent laryngeal nerve were shown. However, in their paper, Kajiyama et al. did not show the thoracic duct in figure one as they mentioned in the figure legend; actually, it is difficult to see it and to detect any topographical relationships based on this figure [1].

Finally, we would like to thank Kajiyama et al. for their interesting work, which has important surgical relevance to esophageal cancer and the prevention of chyloyhorax. Their paper [1] supports our previously reported cadaveric (kentai) research and confirms the fact that anatomy is a keystone of surgery and proper knowledge of the variant anatomy is essential for surgeons.

\section{References}

1. Kajiyama Y, Iwanuma Y, Tomita N, Amano T, Isayama F, Saita M, Ozaki S, Shibamoto M, Kitano H, Uchida T. Relational topographical anatomy between right bronchial artery and thoracic duct. Esophagus. 2014;. doi:10.1007/s10388-014-0450-8.

2. Eid N, Ito Y, Otsuki Y. Thoracic duct relationships to abnormal neurovascular structures in cervicothoracic regions: case study and clinical relevance. Surg Radiol Anat. 2013;35:969-72.

3. Eid N, Otsuki Y. Anomalous bronchial arteries with surgically important relationships to abnormal recurrent laryngeal nerve and thoracic duct. Clin Anat. 2010;23:897-8.

4. Eid N, Ito Y, Otsuki Y. Anomalous bronchial arteries: clinically important relationships to left recurrent laryngeal nerve and thoracic duct. Japanese J Lymphology. 2011;34:53-4. 